\title{
Cervical Spinal Cord Injury: The Correlations of Initial Clinical Features and Blood Gas Analyses with Early Prognosis
}

\author{
P. Myllynen, MD, A. Kivioja, MD, P. Rokkanen, MD, E. Wilppula, MD \\ Department of Orthopaedics and Traumatology, Helsinki University Central \\ Hospital, Topeliuksenkatu 5, SF-00260 Helsinki 26, Finland.
}

\section{Summary}

The predictive values of some early post-traumatic clinical symptoms and signs and laboratory tests on the problems, complications and prognosis of the initial treatment of tetraplegic patients were studied. The study was carried out by scrutinising the files of 54 patients with a cervical spinal cord injury (40 of them complete and 14 incomplete). Most of the patients $(n=43)$ needed ventilatory support, the duration of which depended on the level and completeness of the spinal cord injury. Bradycardia, hypotonia and tachypnoea at admission occurred most frequently in those patients who later developed complications or died. In addition, the frequency of complications correlated with a patient's age, previous diseases and with the height and degree of the spinal cord injury. Tachypnoea on admission forecast the later development of respiratory complications. All 8 patients who died, 5 of them from pneumonia and 3 from pulmonary embolism, had their spinal cord injury at the level C4 to C5 and they were significantly older than those who survived.

Key words: Spinal cord injury; Intensive care; Tetraplegia; Laboratory findings; Prognosis.

Cervical spinal cord injury entails marked disturbances of autonomic, motor and sensory nerve functions which lead to various complications during the initial care (Young, 1979). In particular, respiratory complications (Silver and Gibbon, 1968; Bellamy et al., 1973; McMichan et al., 1980) gastrointestinal bleeding (Kewalramani, 1979; Young, 1979; Masri et al., 1983), deep venous thrombosis and pulmonary embolism (Myllynen et al., 1985) increase the early morbidity and mortality. For optimal treatment it is important to know which patients are most likely to develop complications or even to die. Malone (1984) has found an association between autonomic imbalance, respiratory complications and increased mortality in patients with traumatic tetraplegia.

The purpose of this study was to examine whether initial clinical features and laboratory investigations could forecast the need for respiratory assistance and 
for parenteral nutrition and the risk of further complications in patients with cervical spinal cord injury.

\section{Patients and methods}

The files of 54 patients with acute traumatic cervical spinal cord injury treated in the years 1975 to 1983 at our intensive care unit were studied. The parameters analysed were age, previous diseases, the level and degree of the spinal cord injury, initial blood pressure, heart rate, respiratory rate, blood gas analysis, respirator treatment, tracheostomy, the duration of nasogastric suction and parenteral nutrition. Complications and deaths during early hospital treatment were reviewed.

The degree of the spinal cord injury was graded by giving the value of 1 to patients with some motor function left, 2 to patients with only some sensory function left and 3 to those with a complete spinal cord lesion in the injured segment.

Statistical analyses were performed using Student's t-, chi-square, Pearson's product moment correlation and Spearman's rank correlation tests.

\section{Results}

\section{General description}

There were 48 men and 6 women whose ages ranged from 13 to 73 years with a mean of 36 years. Tetraplegia was complete in 40 and incomplete in 14 patients. One patient had multiple injuries. Most patients had been healthy before the trauma, though 6 of them were chronic alcoholics and 28 were drunk at the time of injury. Traffic accidents and falls during the summer months were the most common trauma mechanisms. All but one of the patients were treated with the Vinke traction device. Eight fractures were operatively reduced and 4 anterior and 6 posterior decompressions were performed. All 18 operations included a spinal fusion.

\section{Radial pulse and blood pressure}

Cervical spinal cord injury caused bradycardia which was treated with atropine in 29 patients $(54 \%)$. The pulse rate at admission was lower in patients with complete tetraplegia $(\mathrm{r}=0.304, \mathrm{p}<0.05)$ and in those who later developed complications or died (Fig. 1).

Hypotonia, which was treated by sympathomimetics, was observed in 20 patients $(37 \%)$ and the need for medication depended in some respect on the level of the injury $(r=0.335, p<0.05)$. The first systolic blood pressure was lower in patients with complete tetraplegia $(r=0.347, p<0.01)$ and also correlated with the number of complications and the risk of death $(\mathrm{p}<0.05)$ (Fig. 2).

\section{Breathing frequency, blood gas analysis and respiratory assistance}

Respiratory rate on admission correlated with the duration of respiratory assistance $(r=0.298 ; p<0.05)$, and in particular, with later respiratory com- 


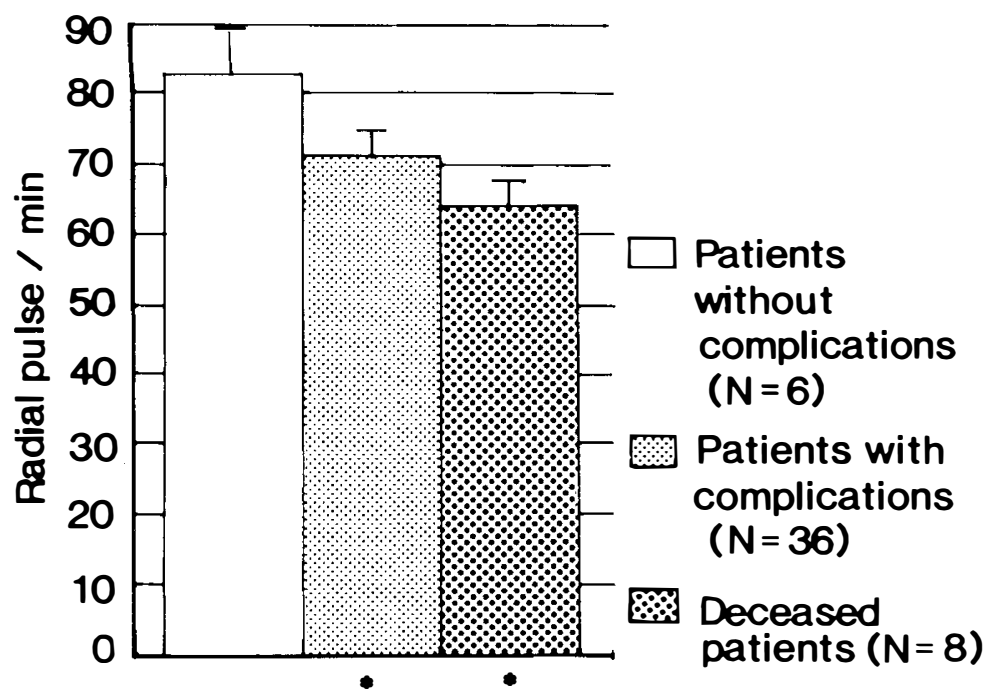

Figure 1 The mean first post-traumatic radial pulse $(\mathrm{min})$ of patients with cervical spinal cord injury. ${ }^{\star} \mathrm{p}<0.05$ as compared with the mean value of the patients without complications.

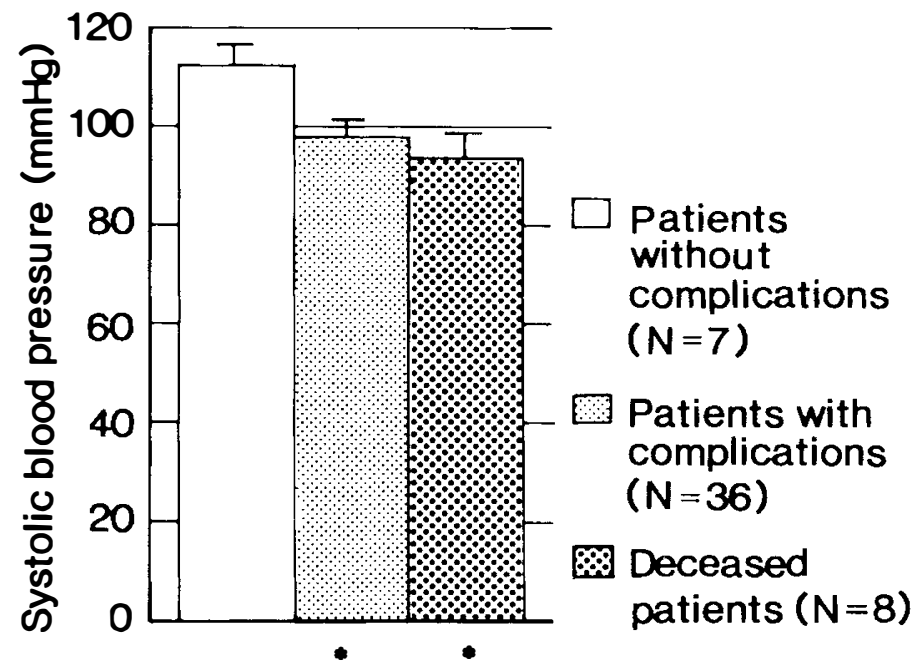

Figure 2 The mean first post-traumatic systolic blood pressure $(\mathrm{mmHg})$ of patients with cervical spinal cord injury.

plications $(\mathrm{r}=0.444, \mathrm{p}<0.001)$. In addition, a respiratory rate on admission was reflected in more numerous complications $(r=0.393, p<0.01)$ and in an increased risk of death $(\mathrm{r}=0.266, \mathrm{p}<0.05)$ during treatment.

Five patients initially had a marked hypoxaemia $\left(\mathrm{pO}_{2}\right.$ below $\left.60 \mathrm{mmHg}\right)$ and 10 patients a moderate hypoxaemia $\left(\mathrm{pO}_{2}\right.$ between 61 and $79 \mathrm{mmHg}$ ). The average $\mathrm{pO}_{2}$ at admission was also below normal, $75 \pm 2 \mathrm{mmHg}$ (mean \pm SEM). However, there was no correlation between the primary $\mathrm{pO}_{2}$ values, later complications and the risk of death. 


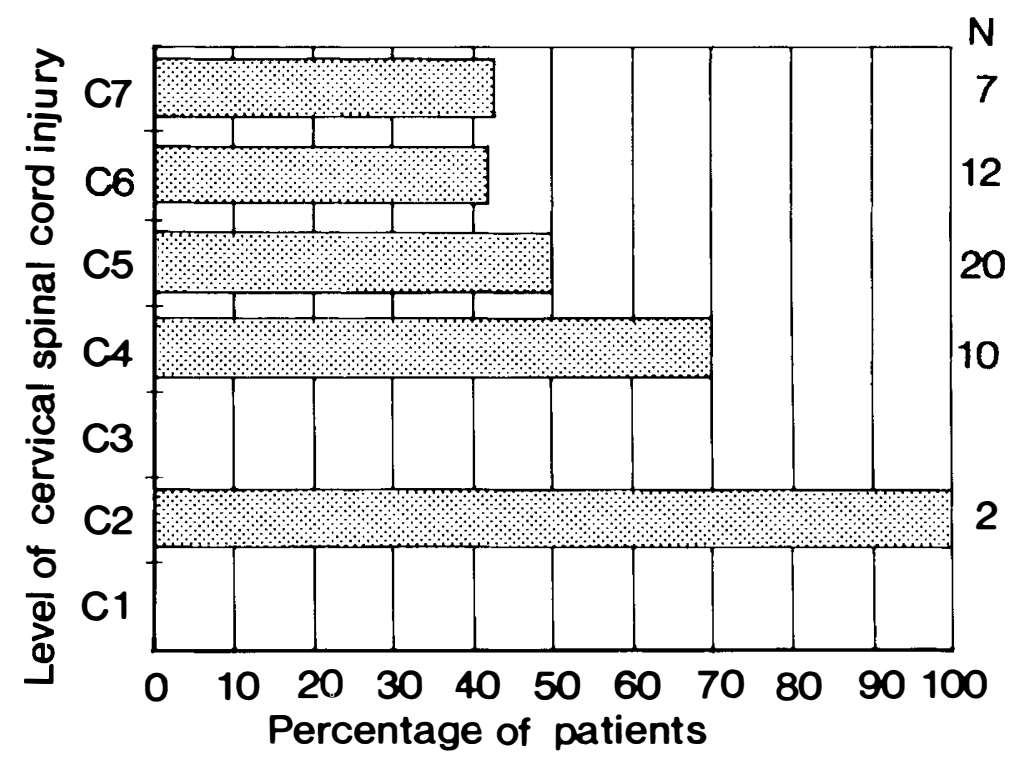

Figure 3 The percentage of patients who needed respirator treatment during intensive care according to the level of the cervical spinal cord injury. $\mathrm{N}=$ number of patients; $\mathrm{C}=$ cervical.

On admission 17 patients hyperventilated $\left(\mathrm{pO}_{2}\right.$ below $35 \mathrm{mmHg}$ ) and 11 patients hypoventilated $\left(\mathrm{pCO}_{2}\right.$ more than $\left.45 \mathrm{mmHg}\right)$. The mean $\mathrm{pCO}_{2}$ value of $39 \mathrm{mmHg}$ was, however, normal. The primary $\mathrm{pCO}_{2}$ value correlated with the height of the spinal cord injury $(r=0.273, p<0.05)$ and with the duration of respirator treatment $(\mathrm{r}=0.265, \mathrm{p}<0.05)$.

The mean blood $\mathrm{pH}$ at admission was at the lower normal limit $(7 \cdot 36 \pm 0 \cdot 01)$ and 21 patients were acidotic according to the first post-traumatic blood gas analyses. The first post-traumatic $\mathrm{pH}$ value correlated inversely with the height of the spinal cord injury $(r=-0.347, \mathrm{p}<0.05)$.

Forty three patients needed ventilatory assistance and tracheostomy was performed on 22 patients. Respirator treatment was begun during the first 6 post-traumatic days and lasted for 17 days on average (from 4 to 30 days). The need for respirator treatment depended on the level $(r=0.317$, $\mathrm{p}<0.05)$ (Fig. 3) and on the completeness $(\mathrm{r}=0.419, \mathrm{p}<0.01)$ of the spinal cord injury but not on the first values of blood pressure, pulse rate or blood gas analysis.

\section{Nasogastric suction and parenteral nutrition}

Forty nine patients needed nasogastric suction for 8.5 days on the average. Parenteral nutrition or fluid therapy was given to all patients for 18 days (from 1 to 110 days). The duration of parenteral nutrition (Fig. 4) correlated with the level of the spinal cord injury $(r=0.418, p<0.01)$. Low primary systolic blood pressure and $\mathrm{pH}$ were more common in those who needed longer parenteral nutrition $(\mathrm{r}=-0.294$ and $-0.282, \mathrm{p}<0.05$, respectively). 


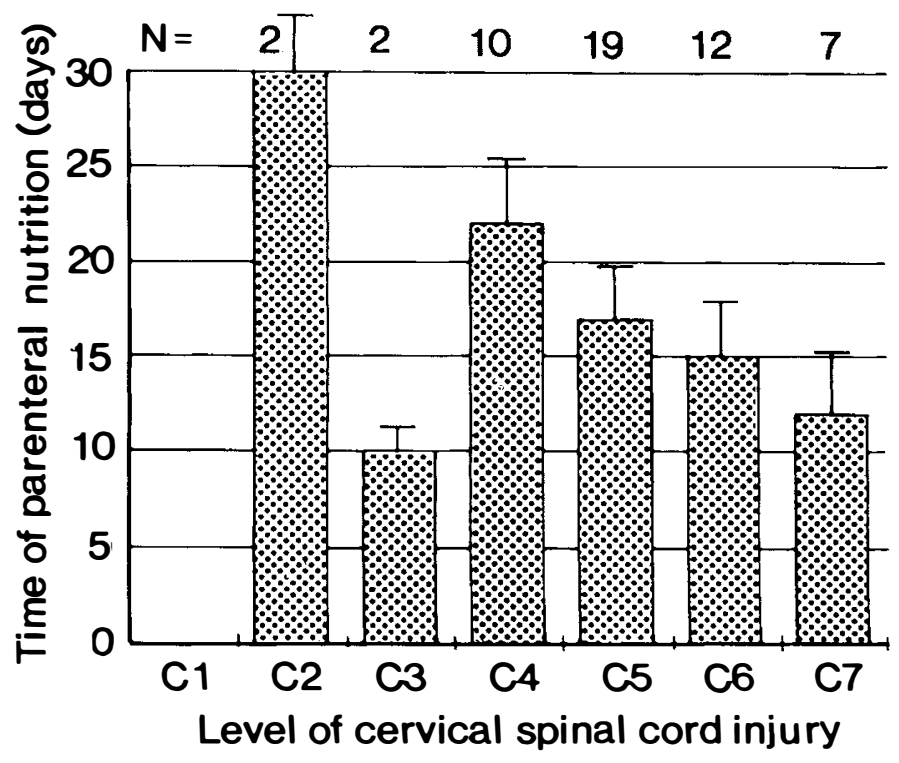

Figure 4 The mean time of parenteral nutrition (days) needed in patients with cervical spinal cord injury. $\mathrm{N}=$ number of patients, $\mathrm{C}=$ cervical.

Table High spinal cord injury. The complications during initial treatment of 54 patients

\begin{tabular}{lrr}
\hline Complication & N & " \\
\hline Urinary infection & 31 & 57 \\
Respiratory infection & 24 & 44 \\
Pulmonary atelectasis & 24 & 44 \\
Gastrointestinal bleeding & 9 & 17 \\
Thromboembolism & 7 & 13 \\
Decubitus & 7 & 13 \\
Cardiovascular complication & 5 & 9 \\
Sepsis & 4 & 8 \\
Acute pancreatitis & 2 & 4 \\
Other minor complications & 16 & 30 \\
\hline
\end{tabular}

\section{Complications}

Patients with a complete spinal cord injury showed significantly more complications during primary care than those who had some sensory or motor function left $(r=0.431, p<0.01)$. In addition, the number of complications during treatment was related to the age of the patients $(r=0.310, p<0.05)$. The most common complications were: urinary tract infection in 31 patients and lung infection or atelectasis in 24 patients (Table). Initial tachypnoea forecast the later development of respiratory complications $(r=0.444, p<0.001)$. Five patients died from pneumonia. Thromboembolism was verified in 7 patients $(13 \%)$ and was the principal cause of death for 3 patients. The average age of the deceased patients, $58 \pm 10$ years (mean \pm SEM), was markedly higher than that of the survivors $(33 \pm 16$ years, $\mathrm{p}<0.001)$. The risk of death depended 


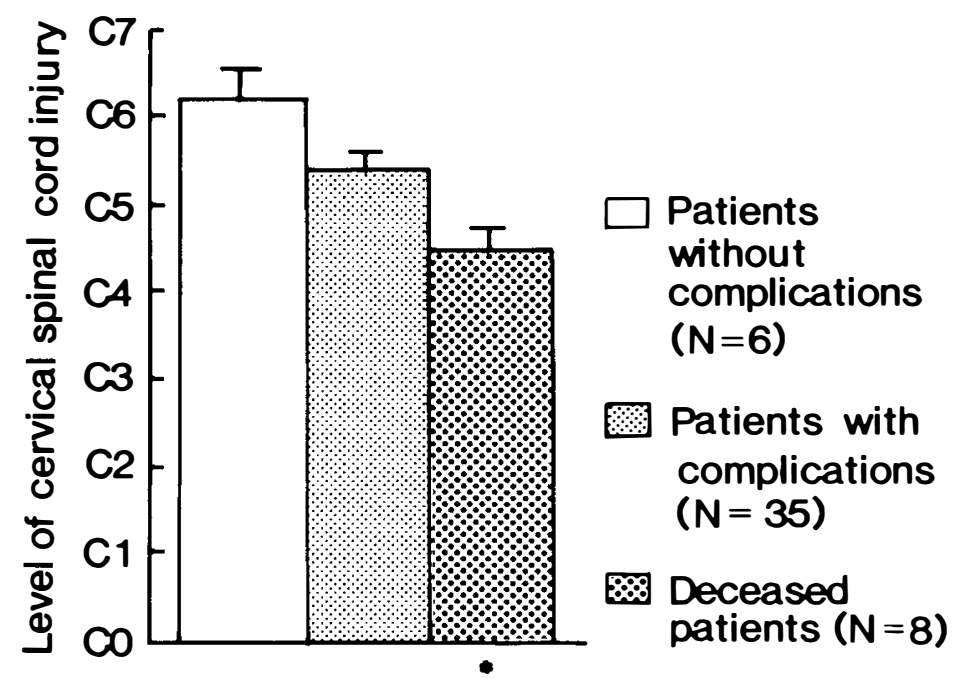

Figure 5 The mean level of cervical spinal cord injury. The patients with or without complications and the dead patients. ${ }^{\star} \mathrm{p}<0.05$ as compared with the patients without complications. $\mathrm{C}=$ cervical.

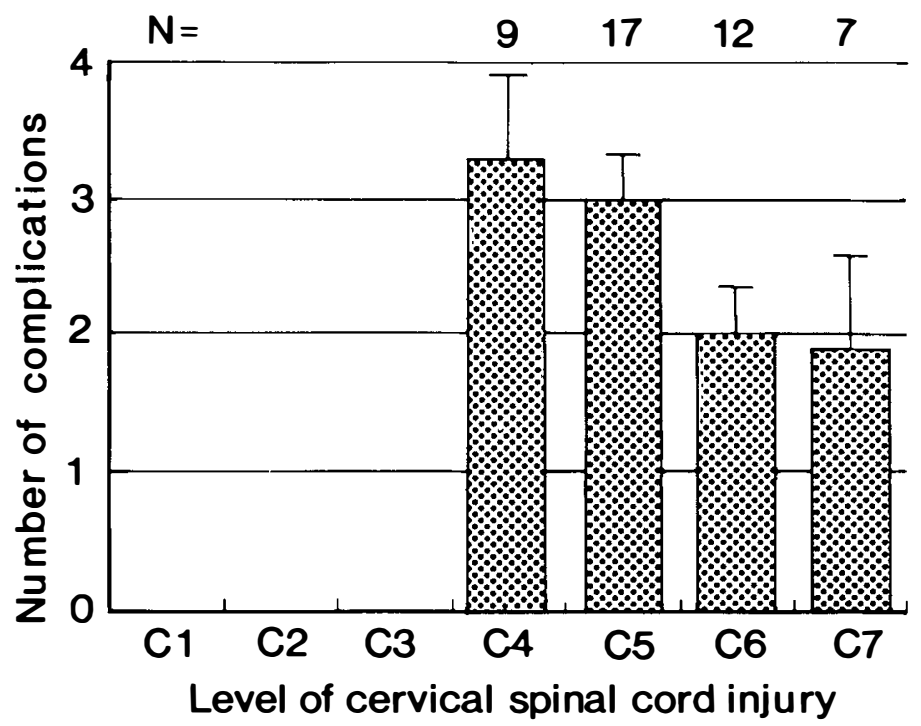

Figure 6 The mean number of complications during intensive care in patients with cervical spinal cord injury. $\mathrm{N}=$ number of patients, $\mathrm{C}=$ cervical.

highly significantly on the number of previous diseases $(r=0.519, \mathrm{p}<0.001)$ and in some respect on the level of the spinal cord injury $(r=0.329, p<0.05)$ (Fig. 5) so that all the deceased patients had their spinal cord injury at the level C4 to C5. Nine patients had gastrointestinal bleeding, 2 patients had acute pancreatitis, 4 had sepsis, 5 had cardiac arrhythmias and 7 had skin problems. The total number of complications was more numerous in those who had an injury at the level C4 to C5 (Fig. 6). 


\section{Discussion}

The difficulties in the primary care of patients with tetraplegia are not only due to motor and sensory paralysis, but also to a great extent to disturbances in vegetative nerve function. Cervical spinal cord injury leads to a total or almost total loss of sympathetic tone below the level of injury, so that vagal innervation from above the injury level dominates (Malone, 1984). Due to the decrease of sympathetic tone the heart rate slows down and blood pressure sinks (McCagg, 1986), respiratory tract secretions change their consistency, the bronchi constrict (McMichan et al., 1980), the bowel tonus changes (McCagg, 1986), possibly gastric acid secretion increases and gastric mucosal circulation decreases (Kewalramani, 1979). The imbalance of the autonomic nerve system becomes stabilised after a week or two. Until then the haemodynamic changes are easily managed through medication.

The aforesaid physiologic disturbances are usually followed by numerous complications during primary treatment. According to the present study certain initial features and laboratory values predict, to some extent, the need for respirator treatment, parenteral nutrition, complications and risk of death. All these predictive factors either describe the severity of the injury or reflect the physiological disturbances associated with it. Our results agree well with the findings of Malone (1984) concerning the relation of the pulse rate and blood pressure to the early prognosis of patients with tetraplegia. However, in contrast to the present outcome, Malone did not find any correlation between age, level of paralysis or respiratory rate and the need for respirator treatment or the risk of death during the early post-traumatic phase. This may be due to his smaller material $(\mathrm{n}=18)$ and to the fact that all older patients ( $>50$ years) were excluded from the study.

The most dangerous early complication is respiratory insufficiency (Bellamy et al., 1973; McMichan et al., 1980) which is a consequence of the almost complete paralysis of the respiratory muscles with the retention of secretions and hypoventilation. This is probably contributed to by the decreased sympathetic activity with an increase in bronchial secretion and constriction. The condition leads to pulmonary atelectasis and often to bronchopneumonia. Therefore patients who have an early post-traumatic tachypnoea, predicting further respiratory complications and the later need for respirator treatment, should be treated by vigorous pulmonary therapy and ventilatory support from the very beginning.

Traumatic high sympathectomy is also followed by paralytic ileus and an increased risk of gastric haemorrhage (Kewalramani, 1979; Masri et al., 1983; Kiwerski, 1986) and other gastrointestinal complications (Young, 1979). To prevent gastrointestinal complications nasogastric suction and parenteral nutrition should be used with wide indications. The long duration of this treatment seems to be, to some extent, predicted by an injury at the fourth and fifth cervical segments, low systolic blood pressure and acidosis at admission. Actually, the two latter prognostic signs are conjoined to the high level of the spinal cord injury and only indicate the greater influence of those injuries on circulatory and respiratory functions. 


\section{Conclusion}

A tetraplegic patient can have several different complications during early treatment in the intensive care unit which inevitably increases early mortality. Therefore it is important to find the patients at high risk. The present study shows that the age and previous diseases of the patient, the level and degree of spinal cord injury, and blood pressure, heart rate and respiratory frequency on admission can have a prognostic value when estimating the early post-traumatic course of patients with cervical spinal cord injury.

\section{References}

Bellamy R, Pitts FW, Stauffer ES 1973 Respiratory complications in traumatic quadriplegia. Analysis of 20 years' experience. Fournal of Neurosurgery 39:596-600.

KEWALRAMANI LS 1979 Neurogenic gastroduodenal ulceration and bleeding associated with spinal cord injuries. Fournal of Trauma 19:259-265.

KIWERSKI J 1986 Bleeding from the alimentary canal during the management of spinal cord injury patients. Paraplegia 24:92-96.

MALONE PR 1984 Early prognosis in traumatic tetraplegia. Injury 16:2-5.

MASRI WEI, Cochrane P, SiLver JR 1983 Gastrointestinal bleeding in patients with acute spinal injuries. Injury 14:162-167.

MCCAGG 1986 Postoperative management and acute rehabilitation of patients with spinal cord injuries. Orthopaedic Clinics of North America 17:171-181.

MCMichan JC, Michel L, Westbrook PR 1980 Pulmonary dysfunction following traumatic quadriplegia. Recognition, prevention, and treatment. F $A M A$ 243:528-531.

Myllynen P, Kammonen M, RokKanen P, et al. 1985 Deep venous thrombosis and pulmonary embolism in patients with acute spinal cord injury: A comparison with non-paralyzed patients immobilized due to spinal fractures. Fournal of Trauma 25:541-543.

Silver JR, GibBon NOK 1968 Prognosis in tetraplegia. British Medical fournal 4: 79-83.

YouNG JS 1979 Spinal cord injury: Associated general trauma and medical complications.

Thompson RA and Green RJ (eds) Advances in Neurology, vol 22, Raven Press, New York, pp 255-260. 